\title{
Effects of Applied Strain and Subsequent Heat Treatment at Intermediate Temperature on Mechanical Properties of a Thin Plate Ti-51 at\% Ni Shape Memory Alloy*1
}

\author{
Hiromasa Semba ${ }^{1, * 2}$, Nagatoshi Okabe ${ }^{1}$, Toru Yamaji ${ }^{1, * 3}$, \\ Keisuke Okita ${ }^{1, * 2}$ and Kiyoshi Yamauchi ${ }^{2}$ \\ ${ }^{1}$ Department of Mechanical Engineering, Ehime University, Matsuyama 790-8577, Japan \\ ${ }^{2}$ Biomedical Engineering Research Organization, Tohoku University, Sendai 980-8575, Japan
}

\begin{abstract}
Attempts have been made to develop new types of seismic devices using shape memory alloys. They are a single-stage bellows which are processed from thin-walled tubes by employing the rubber bulge method and then are annealed at $400^{\circ} \mathrm{C}$ for the shape-memory treatment. As strain distribution is induced on the bulged part due to the process, it is significant to know the effects of pre-strain and the subsequent heattreatment on the mechanical properties of the material for tube when designing the bellows shapes for the seismic structures. Thus, tensile tests and thermal analysis were conducted before and after the heat treatment on rectangular specimens cut from the tubes.

In this paper, the oxidization method was first attempted to observe distinctively both regions of the stress induced martensite (SIM) transformation and twin deformation generated while applying strain to the specimens. It became clear that the micro-structure in the SIM area had a changed $\mathrm{R}$ phase from the austenite phase at room temperature after being annealed at $400^{\circ} \mathrm{C}$. From the experimental and analytical results for the specimens, the mechanical behavior was classified broadly into two conditions as follows: (1) the mechanical behavior can be formulated on the basis of a series-model consisting of areas of both $\mathrm{R}$ phase and austenite considering the area-ratio of these phases until the SIM transformation has expanded over the whole specimen, and (2) after that, the mechanical behavior can be formulated using the exponential function for the applied strain as a parameter.
\end{abstract}

(Received August 22, 2005; Accepted November 22, 2005; Published March 15, 2006)

Keywords: shape memory alloy, applied strain, heat treatment, titanium-nickel alloy, stress-induced martensite transformation, differential scanning calorimeter, rhombohedral phase, austenite phase

\section{Introduction}

It is known that the transformation (and reverse-transformation) temperatures and mechanical properties of shape memory alloys (SMAs) depend significantly on how the cold working and heat-treatments are applied to them. Entangling of the propagated dislocations due to slip deformation, precipitation due to heat-treatment and residual stress field around dislocations and/or precipitations are seen as major causes of changing their properties. Especially on SMAs of Ti-Ni 2 system alloy that is most known in SMA's, heattreatment at their recrystallization temperature and ageing treatment at intermediate temperature are typical ways of changing their mechanical properties. ${ }^{1-4)}$

We have been developing a new type of seismic protection elements for preventing buildings and social infrastructures from being destroyed by big earthquakes or other strong impacts. They are made of shape memory alloy and formed into bellows structure. They are called SMA single-stage bellows, ${ }^{5,6)}$ expected to have synergistic effects of the material $^{7,8)}$ and the structure. The forming procedure of SMA single-stage bellows is as follows. Thin-walled TiNi tubes subjected to cold working are prepared as as-received samples. They are first annealed above their recrystallization temperature to improve their workability. Next specific part of the tube is bulged using the rubber bulge method by controlling compressive displacement. After that, they are

\footnotetext{
${ }^{* 1}$ This Paper was Originally Published in Japanese in J. Jpn. Inst. Met. 69 (2005) 587-595.

${ }^{* 2}$ Graduate Student, Ehime University

${ }^{*} 3$ Graduate Student, Ehime University, Present address: Mitsubishi Electric Corporation
}

also annealed at intermediate temperature for shape memory treatment.

It is easily imagined that bulge-formed SMA bellows have strain distribution in them. So in practical use, it is very important to know the mechanical properties and the phase corresponding to the strain (or shape) for FEM analysis on dynamic behavior of SMA bellows and for their shape design.

Thus, in this research, influences of pre-strain and subsequent heat-treatment at intermediate temperature on mechanical properties of thin plate of $\mathrm{Ti}-51$ at $\% \mathrm{Ni}$ shape memory alloy were investigated. Here, pre-strain is the maximum applied strain under a tensile test. After applying the maximum strain, each specimen was unloaded and removed from the apparatus. After that they were annealed below recrystallization temperature without constraint. Tensile test was also carried out for them, and the relationship between stress and strain was formulated as a parameter of pre-strain.

In this paper, it is first confirmed through our experiment that for the rectangular thin plate specimens we can easily distinguish the area having experienced stress-induced martensite transformation from the area not having experienced it during tensile tests by using oxidization method. Next the influence of pre-strain and subsequent heat-treatment on their microstructural phases is clarified by thermal analysis. From the tensile test results of the specimens after annealing at intermediate temperature, it is clarified that the mechanical behavior changes significantly depending on the condition of pre-strain. Finally the relation formula between stress and strain is obtained with a parameter of pre-strain, which offers knowledge of basic data for processing and/or designing SMA single-stage bellows. 


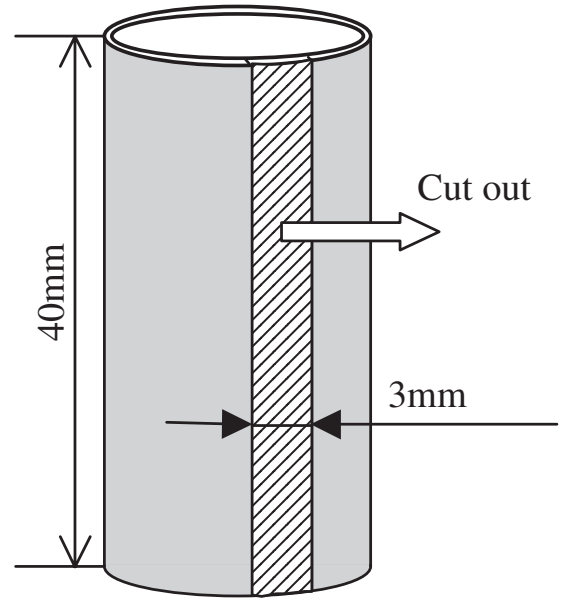

Fig. 1 Test piece for tensile test.

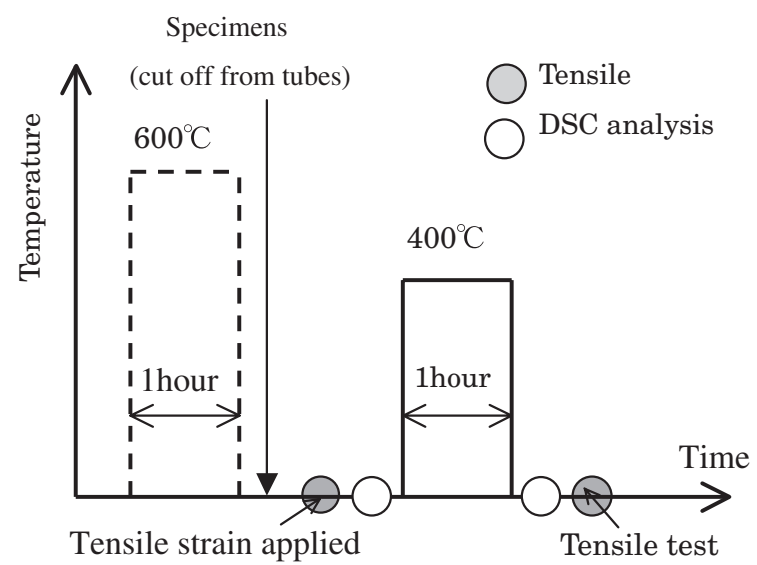

Fig. 2 Test chart of heat treatments and tensile tests.

\section{Experiment}

\subsection{Alloy and specimens}

The study was carried out for Ti-Ni binary alloy, whose composition was Ti-51 at\% Ni. As-received samples were tubes of $10.1 \mathrm{~mm}$ in outer diameter and $0.33 \mathrm{~mm}$ in thickness, with $20 \%$ cold working. They were first cut in $40 \mathrm{~mm}$ length and annealed at $600^{\circ} \mathrm{C}$ for $1 \mathrm{~h}$ in an electric furnace and then water quenched. These heat-treated samples were then cut in about $3 \mathrm{~mm}$ width as shown in Fig. 1. The rectangular thin plate specimens thus obtained were used for the subsequent tests.

\subsection{Experimental Procedure}

The process of experiment is shown in Fig. 2. The rectangular thin plate specimen mentioned above was first immersed in boiled water for $1 \mathrm{~min}$ for ensuring to be in austenite phase at room temperature. Then, $10 \mathrm{~mm}$ lengths from both ends of the specimen were fixed in the chucks of the apparatus ${ }^{9)}$ as shown in Fig. 3. Gauge length of the specimen was set to $20 \mathrm{~mm}$. Tensile tests were carried out at the speed of $5 \% / \mathrm{min}$ and at room temperature. After the tension was applied to the specimen until the predefined maximum strain, it was unloaded at the same speed as the tension and then removed from the apparatus. After that they

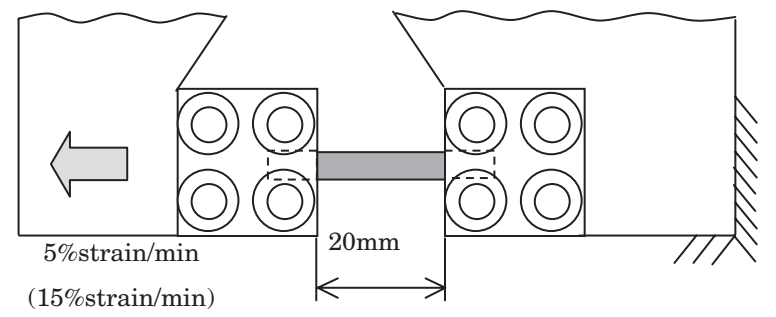

Fig. 3 Experimental set-up for tensile tests.

were annealed at $400^{\circ} \mathrm{C}$ for $1 \mathrm{~h}$ in the electric furnace at atmospheric pressure and water quenched. They were then immersed in boiled water again, and the tensile tests were also carried out until fracture using the same apparatus shown in Fig. 3.

In addition, other specimens with various pre-strains were analyzed on their microstructural phase state using differential scanning calorimetry (RIGAKU DSC8230) for small pieces cut from the area having experienced stress-induced martensite transformation and the area not having experienced it.

\section{Results and Discussion}

\subsection{Visualization of stress-induced martensite trans- formation and twin deformation}

As Ti-Ni alloy is easy to be oxidized at high temperature, the heat-treatment at high temperature is commonly carried out in high vacuum or in inactive gas. However, we utilized the oxidization method for visualizing the stress-induced martensite transformation and subsequent twin deformation.

The surface of a well polished TiNi was oxidized through the heat-treatment at $600^{\circ} \mathrm{C}$ for $1 \mathrm{~h}$, resulting in its color turned from metallic color to charcoal gray. Microstructural phase of the material after the heat-treatment was in austenite phase at room temperature as shown in Table 1. Therefore it is imagined that the specimens show the stress-induced martensite transformation and reverse transformation when loaded and unloaded above austenite finish temperature $\left(A_{\mathrm{f}}\right)$.

A rectangular thin plate specimen was loaded at the speed of $15 \% \mathrm{strain} / \mathrm{min}(3.0 \mathrm{~mm} / \mathrm{min})$ until $12 \%$ strain along the curve paths in stress-strain diagram and then unloaded as shown in Fig. 4. Temperature changes and temperature distribution of the specimen were measured in this sequence process by thermoviewer (NEC Sanei/thermo-tracer TH7102). Additionally at the same time, appearances of the specimen were taken using a digital camera at some strains.

The temperature distribution diagram and a photograph of the specimen at point A (7\% strain) are shown in Fig. 5 and at point B (11\% strain) in Fig. 6, respectively. Stress-plateau between $C$ and $D$ in Fig. 4 indicates the region where the stress-induced martensite transformation and subsequent twin deformation must have occurred. From the temperature

Table 1 Transformation and reverse transformation temperatures for annealing at $600^{\circ} \mathrm{C}$ for $1 \mathrm{~h}$.

\begin{tabular}{ccccc}
\hline & $M_{\mathrm{f}}$ & $M_{\mathrm{s}}$ & $A_{\mathrm{s}}$ & $A_{\mathrm{f}}$ \\
\hline Temperature & $-49^{\circ} \mathrm{C}$ & $-30^{\circ} \mathrm{C}$ & $-5^{\circ} \mathrm{C}$ & $6^{\circ} \mathrm{C}$ \\
\hline
\end{tabular}




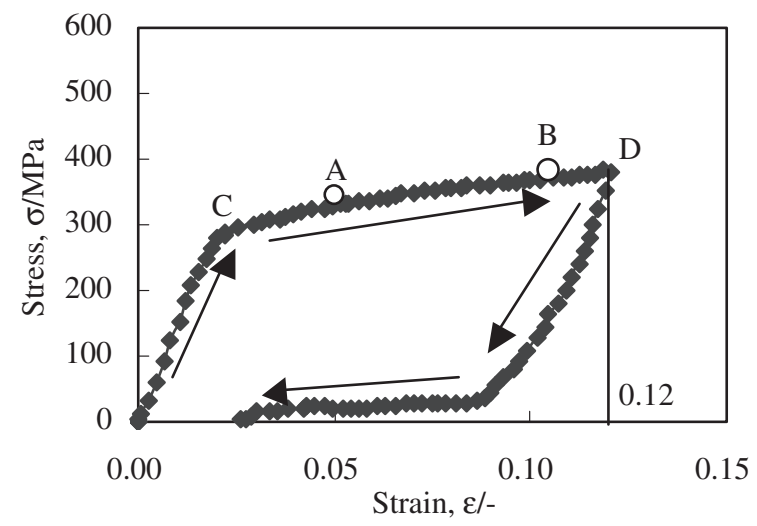

Fig. 4 Load and unload procedure on stress-strain curve.

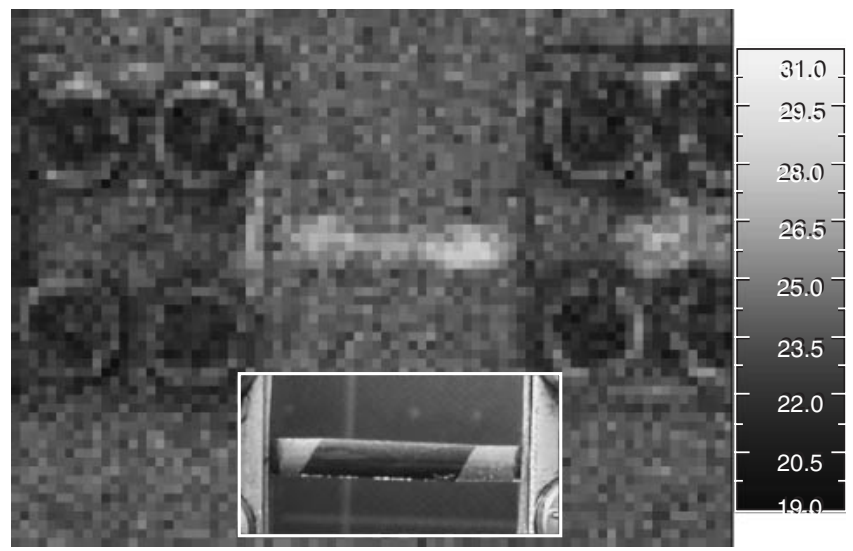

Fig. 5 Thermal view on point A compared with appearance of the specimen.

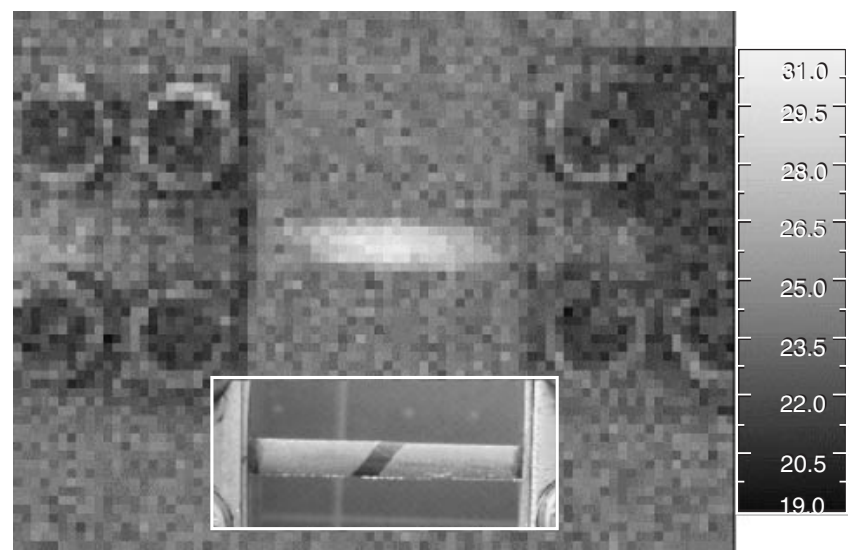

Fig. 6 Thermal view on point B compared with appearance of the specimen.

distributions shown in Figs. 5 and 6, it is clearly imaginable that exothermal reaction of prominent feature of stressinduced martensite transformation was generated as a local temperature rise and that the area of high temperature moved. This suggests the stress-induced martensite transformation to propagate like Luder's deformation.

It is interesting to note that position of exothermal area on stress-induced martensite transformation corresponds well to discoloration (from thick-gray to thin-gray) area in the

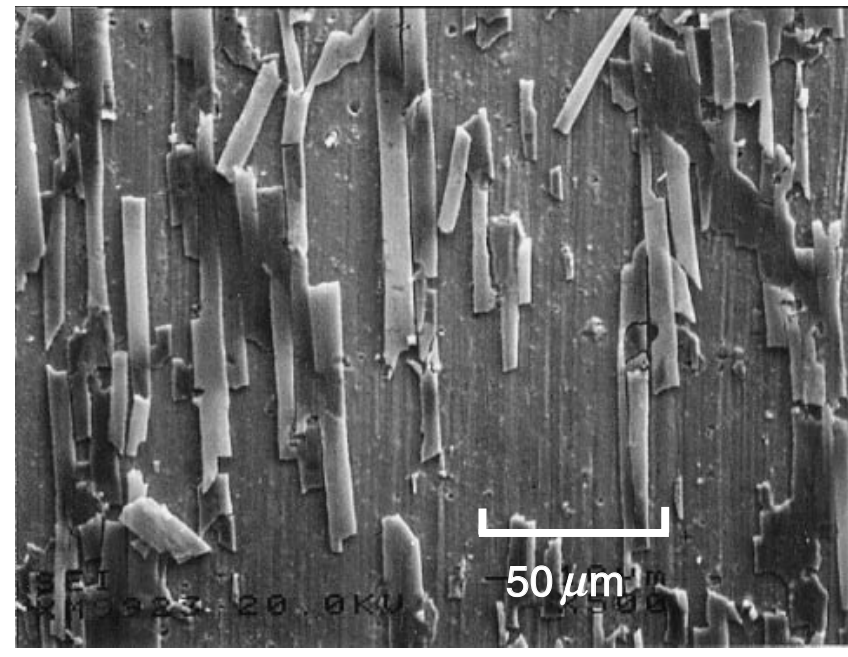

Fig. 7 SEM image of the surface.

specimen. From this evidence, it is distinctly confirmed that stress-induced martensite transformation and subsequent twin deformation occur almost at the same time and that the discoloration area of the specimen suggests that the stress-induced martensite transformation and subsequent twin deformation have been experienced. Moreover, it became clear that stress-induced martensite transformation and twin deformation occur at 45 degree against the tensile, namely the shear direction, and proceed from chuck sides to the central point of the specimen.

It is considered that the discoloration relates to the oxidized coating generated on the surface of the specimen when annealed at high temperature and at atmospheric pressure. It was confirmed by SEM image (shown in Fig. 7) that the discoloration resulted from oxidized coating that peeled off from the substrate due to the deformation difference between the coating and the substrate.

\subsection{Mechanical behavior and thermal analysis after high temperature heat-treatment}

Tensile test of each rectangular thin plate specimen annealed at $600^{\circ} \mathrm{C}$ for $1 \mathrm{~h}$ was carried out loading the specimen to the predetermined strain (maximum applied strain $\varepsilon_{\max }$ ) and unloading it soon. Stress-strain diagrams obtained are shown in Fig. 8. From this figure, it was confirmed that all specimens followed almost the same path on tensile process. Although the stress-plateau has a little slope against strains, the curve shows a deformation behavior of austenite phase.

The thermal analysis results are shown in Fig. 9. For the analysis, DSC specimens were cut out from both the stressinduced martensite transformation experienced area (called SIM area) and the not-experienced area (called noSIM area). Thermal analysis were carried out on the temperature descending process from 100 to $-80^{\circ} \mathrm{C}$ (or $-70^{\circ} \mathrm{C}$ ) and then on the temperature ascending process (the reveres process) at temperature changing rate of $5^{\circ} \mathrm{C} / \mathrm{min}$. Vertical axis in the figure indicates heat flow per unit mass.

From the results in temperature descending process, it was found that martensite start temperature $\left(M_{\mathrm{S}}\right)$ hardly depends on whether the specimen has experienced stress-induced 




Fig. 8 Stress-strain curves for different maximum strain $\varepsilon_{\max }$ after annealing at $600^{\circ} \mathrm{C}$ for $1 \mathrm{~h}$.

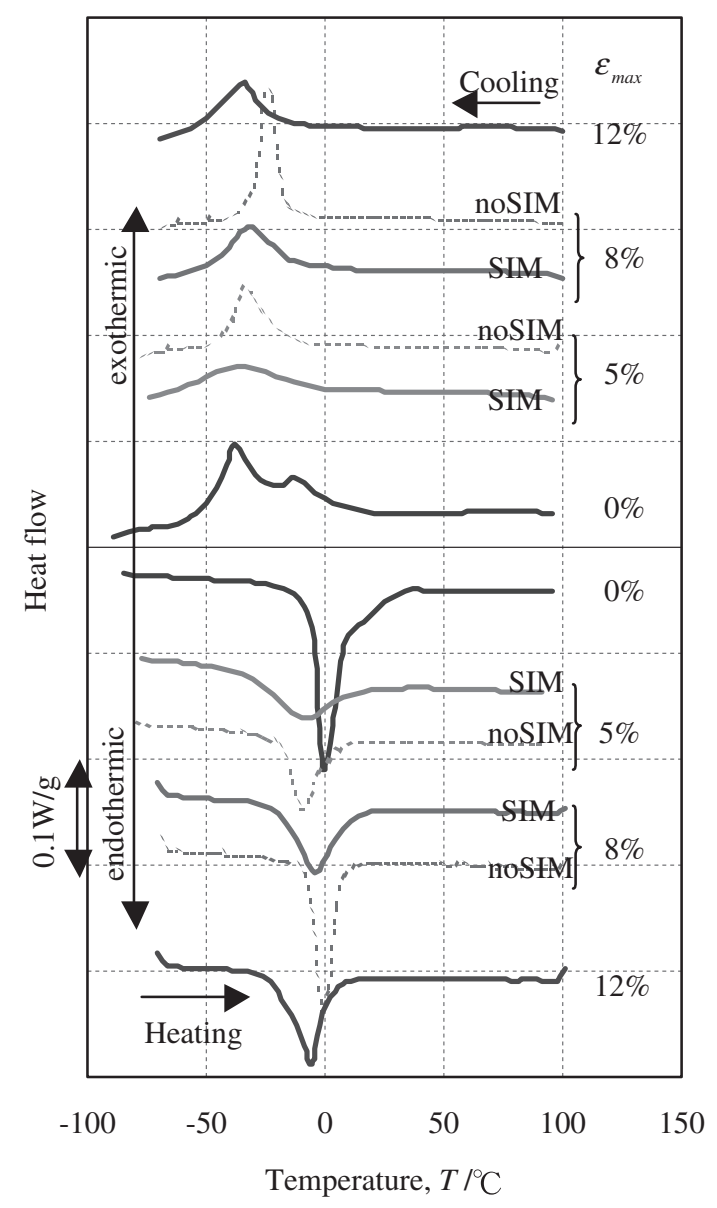

Fig. 9 DSC result of the specimens after $600^{\circ} \mathrm{C} \times 1 \mathrm{hWQ}$ for different applied strain in descending and ascending temperatures.

martensite transformation or not, until reaching the $12 \%$ prestrain, when the whole tensile specimen have finished stressinduced martensite transformation. However martensite finish temperature $\left(M_{\mathrm{f}}\right)$ shifts to a lower value in the specimens cut out from SIM area and the curves have dull peaks. On the other hand, the DSC curves of noSIM have sharper peaks than SIM's case. This trend is found also in the temperature ascending process. It is considered that there are more fixed dislocations and wider internal stress fields in SIM area than in noSIM area.

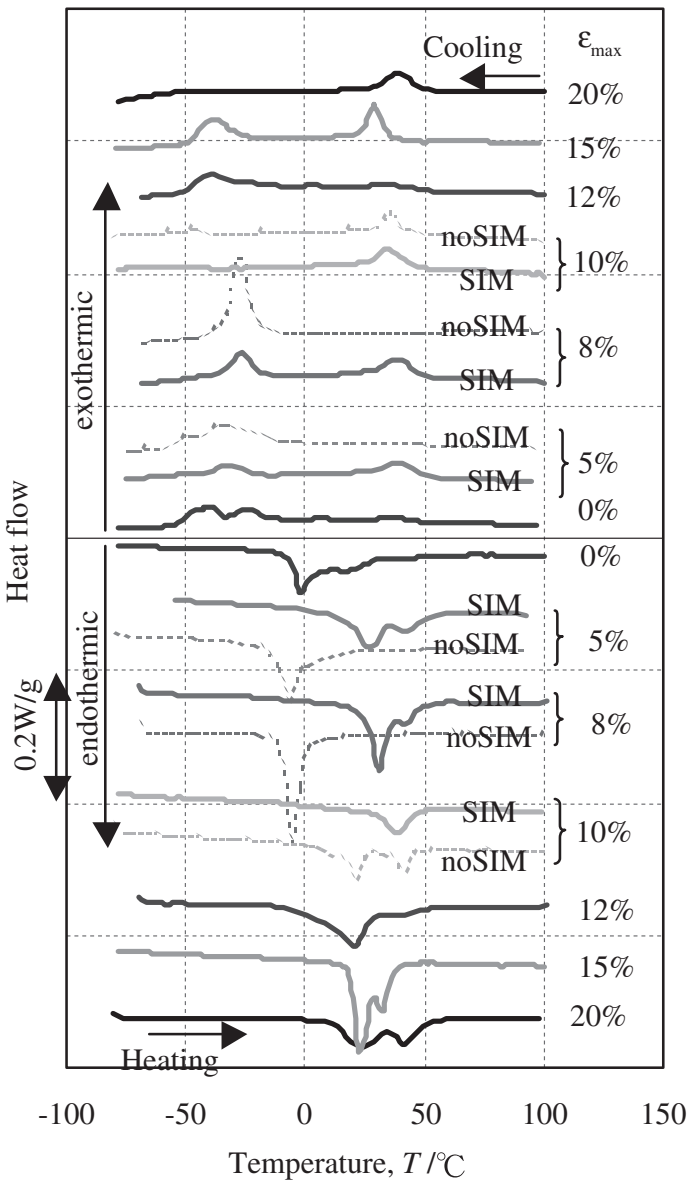

Fig. 10 DSC result of the specimens after $400^{\circ} \mathrm{C} \times 1 \mathrm{hWQ}$ for different applied strain in descending and ascending temperatures.

In addition, it is recognized that the microstructural phase of the material is in austenite state without relating to whether it is of SIM area or of noSIM area in the case below $12 \%$ prestrain.

\subsection{Thermal analysis results of the specimens with subsequent heat-treatment at intermediate temper- ature as well as with pre-strain by tension}

DSC thermal analysis results of the specimens after annealing at $400^{\circ} \mathrm{C}$ for $1 \mathrm{~h}$ after pre-strain are shown in Fig. 10. DSC curves with various pre-strains as a parameter are indicated in this figure. The results for SIM area are indicated as solid lines, while noSIM's are indicated as dashed lines only on results with pre-strain of 5,8 and $10 \%$. As for the sampling of specimens, (1) concerning the specimen for SIM area, they were basically cut out to about $3 \mathrm{~mm}$ length from the border line between SIM area and noSIM area to chuck side, while (2) concerning the specimen for noSIM area, they are cut out to about $3 \mathrm{~mm}$ from $2 \mathrm{~mm}$ apart from the border line. However concerning the specimen of $\varepsilon_{\max }=10 \%$, as it was hard to sample in the above mentioned way, specimen was cut out between two borderlines.

From the DSC results for SIM areas as shown in Fig. 10, it is found that a new peak with respect to exothermal reaction appears around 40 after $400^{\circ} \mathrm{C}$ heat-treatment. As a result, each DSC curve for SIM area has generally 4 peaks during 


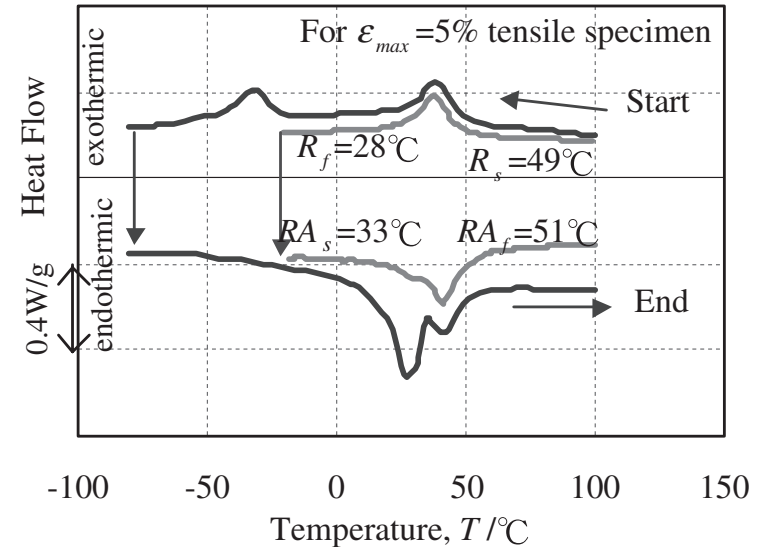

Fig. 11 DSC result of $5 \%$ tensile specimen for different temperature ranges between 100 and $-70^{\circ} \mathrm{C}$, and between 100 and $-20^{\circ} \mathrm{C}$.

the temperature descending and ascending processes. In order to investigate the phases corresponding to these peaks, the same specimen with pre-strain $\varepsilon_{\max }=5 \%$ as shown in Fig. 10 was tested again by changing the lowest temperature in temperature descending process. The lowest temperature in the process was set to $-20^{\circ} \mathrm{C}, 1^{\circ} \mathrm{C}$ higher than the phasestart-temperature in lower temperature. From the DSC result shown in Fig. 11, it is found that the peak in Fig. 10 does not exist in Fig. 11. Judging from this fact and small temperature hysteresis in this phase, the phase corresponding to this peak is considered to be a rhombohedral phase (R-phase). Therefore it is considered that the peaks in lower temperature in temperature descending process and that in temperature ascending process correspond to martensite phase and austenite phase, respectively.

Focusing on DSC results for noSIM area for $\varepsilon_{\max }=5,8$, $10 \%$, the curves for noSIM area of $\varepsilon_{\max }=5,8 \%$ are different from the curves corresponding to their SIM areas, while the curve of noSIM area of $\varepsilon_{\max }=10 \%$ is similar to the curve of SIM area's. It is considered that these results are caused by difference of internal stress field and/or amount of fixed dislocations between SIM area and noSIM area. For specimens of $\varepsilon_{\max }=5,8 \%$, there are great differences in expanse of internal stress field and/or amount of fixed dislocations, but for specimen of $\varepsilon_{\max }=10 \%$, there is scarcely any difference. Thus, R-phase can appear even in the area of the oxidized coating not peeled off.

Summarizing these results, R-phase absolutely appears in SIM area after $400^{\circ} \mathrm{C}$ heat-treatment, and the microstructural phase becomes R-phase at room temperature when it is cooled from the temperature above $50^{\circ} \mathrm{C}$. Even in the case that the pre-strain $\varepsilon_{\max }$ is below $12 \%$, which is considered to reach the strain for finishing the propagation of stressinduced martensite transformation over the whole specimen, R-phase can appear over the whole specimen.

\subsection{Mechanical properties of the specimens with sub- sequent heat-treatment at intermediate temperature as well as with pre-strain}

Figure 12 shows stress-strain curves of specimens annealed at $400^{\circ} \mathrm{C}$ for $1 \mathrm{~h}$ with a parameter of pre-strain $\varepsilon_{\max }$. The curve of the specimen without pre-strain is indicated as

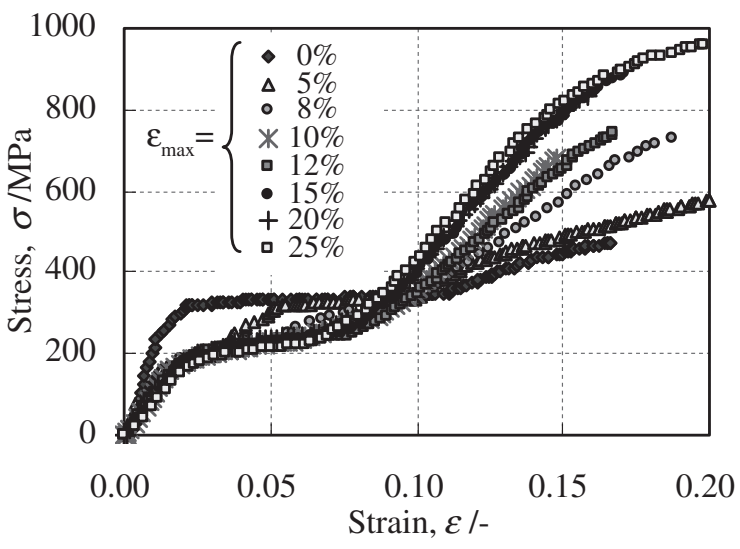

Fig. 12 Stress-strain curves for different maximum strain $\varepsilon_{\max }$ after annealing at $400^{\circ} \mathrm{C}$ for $1 \mathrm{~h}$.

" $0 \% "$ of $\varepsilon_{\max }$ for comparison.

From the figure, it is found that mechanical behavior of the specimen of $\varepsilon_{\max }=0 \%$ after $400^{\circ} \mathrm{C}$ heat-treatment is similar to that of before $400^{\circ} \mathrm{C}$ heat-treatment, that is, stress-induced martensite transformation was recognized in both specimens. On the other hand, comparing mechanical behavior before $400^{\circ} \mathrm{C}$ heat-treatment with after $400^{\circ} \mathrm{C}$ heat-treatment except $\varepsilon_{\max }=0 \%$, there is a great difference in each pre-strain specimen.

It is considered that this concerns R-phase as mentioned before. Additionally, it is found that the stress-strain curves of the specimens with pre-strain $\varepsilon_{\max }$ below $12 \%$ have highly specific behaviors as seen in Fig. 12 and that the behaviors with pre-strain $\varepsilon_{\max }$ above $12 \%$ are similar to the deformation behavior of material in martensite.

Therefore, the stress-strain relation of this material with a parameter of pre-strain $\varepsilon_{\max }$ is discussed in the following section in two cases of $\varepsilon_{\max } \leq 12 \%$ and $\varepsilon_{\max }>12 \%$.

3.4.1 Mechanical properties in the case of $\varepsilon_{\max } \leq 12 \%$

First, mechanical properties of the specimens with prestrain $\varepsilon_{\max }$ below $12 \%$ are discussed. As it is recognized that stress-induced martensite transformation and twin deformation propagate along the longitudinal direction with regularity, a mechanical simulation model of the specimen can be built as a series combined model of SIM area and noSIM area. Although stress-induced martensite transformation actually progress from both fixed ends to the central point of the specimen, a series combined model with only 2 elements is used for simplification as shown in Fig. 13. Here, one element is R-phase material and the other is austenite phase material.

In this model, both volume fractions of R-phase and austenite phase are defined as $p$ and $1-p$, respectively. Considering the model of applied tensile stress $\sigma$, both incremental strains of R-phase element and austenite phase element are defined as $\Delta \varepsilon_{\mathrm{R}}$ and $\Delta \varepsilon_{\mathrm{A}}$, respectively when the tensile stress increases by $\Delta \sigma$. When the tensile stress is increased by $\Delta \sigma$, increment strain of the whole model $\Delta \varepsilon$ becomes $\Delta \varepsilon=p \Delta \varepsilon_{\mathrm{R}}+(1-p) \Delta \varepsilon_{\mathrm{A}}$.

Both static compliances of austenite phase element and of $\mathrm{R}$-phase element are expressed as the inverse of their rigidity $\left(E_{\mathrm{A}}, E_{\mathrm{R}}\right)$, respectively. 


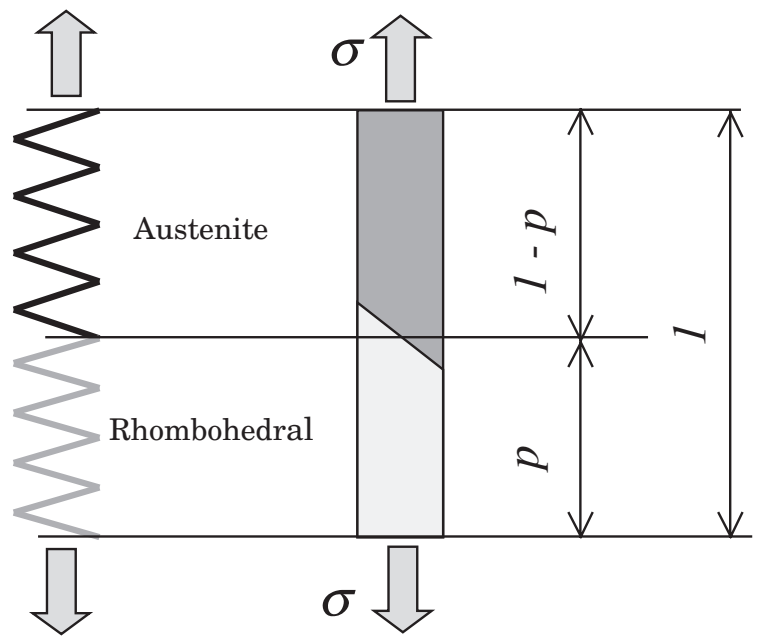

Fig. 13 Series combined model of two phases.

$$
\begin{aligned}
& \left(E_{\mathrm{R}}\right)^{-1}=\frac{d \varepsilon_{\mathrm{R}}}{d \sigma} \\
& \left(E_{\mathrm{A}}\right)^{-1}=\frac{d \varepsilon_{\mathrm{A}}}{d \sigma}
\end{aligned}
$$

As the total strain of the whole model is sum of each product of each phase element strain and the corresponding volume fraction, the following expression is obtained.

$$
\varepsilon(\sigma)=\int d \varepsilon=\int\left\{p \frac{\partial \varepsilon_{\mathrm{R}}}{\partial \sigma}+(1-p) \frac{\partial \varepsilon_{\mathrm{A}}}{\partial \sigma}\right\} d \sigma
$$

Equation (3) is then expanded to eq. (4) as a function of the stress $\sigma$.

$$
\varepsilon(\sigma)=p \varepsilon_{\mathrm{R}}(\sigma)+(1-p) \varepsilon_{\mathrm{A}}(\sigma)
$$

On the other hand, the relational expression between stress and strain of a shape memory alloy can be generally classified into 2 stages according to strain (or stress). In the first stage, elastic deformation is dominant. But in the next stage, twin deformation is dominant. Therefore, the flow stress $\sigma$ during tensile process is approximated as a linear function in the first strain range, and is approximated as an exponential function in the second strain range by eq. (5) or eq. (6) as follows

$$
\begin{gathered}
\sigma=E \varepsilon \\
\sigma=\left(\sigma_{\max }-\sigma_{y}\right)\left[1-\exp \left\{-\left(\frac{\varepsilon-\varepsilon_{\mathrm{el}}}{\varepsilon_{\mathrm{ip}}}\right)^{n}\right\}\right]+\sigma_{y} \\
\left(\varepsilon \geq \varepsilon_{\mathrm{el}}\right)
\end{gathered}
$$

where various parameters are defined in stress-strain diagram shown in Fig. 14. E indicates Young's modulus of a material in the range of elastic strain. $\sigma_{\max }$ and $\sigma_{y}$ indicate saturated stress and yield stress in elastic strain region, respectively. $\varepsilon_{\mathrm{el}}$ indicates strain limit of elastic region, $\varepsilon_{\mathrm{el}}+\varepsilon_{\mathrm{ip}}$ means the strain corresponding to the inflection point of the stressstrain curve.

Thus, the strain $\varepsilon$ in the two ranges is given in the following expressions as a function of the stress $\sigma$.

$$
\varepsilon=\frac{\sigma}{E} \quad\left(0 \leq \varepsilon \leq \varepsilon_{\mathrm{el}}\right)
$$

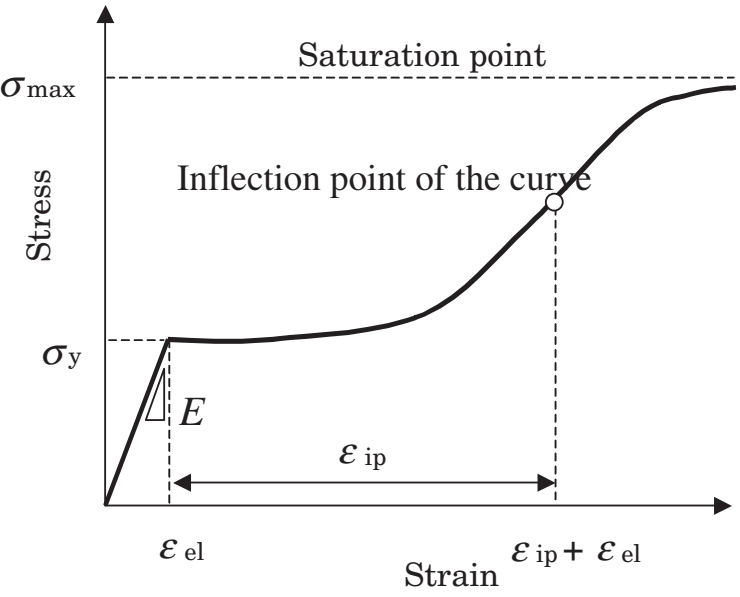

Fig. 14 Definition of deformation characteristics in Stress-strain curves.

$$
\varepsilon=\varepsilon_{\mathrm{ip}}\left[-\ln \left\{1-\frac{\sigma-\sigma_{y}}{\sigma_{\max }-\sigma_{y}}\right\}\right]^{\frac{1}{n}}+\varepsilon_{\mathrm{el}} \quad\left(\varepsilon \geq \varepsilon_{\mathrm{el}}\right)
$$

Mechanical behavior of R-phase element and austenite phase element is expressed as eqs. (7) and (8), respectively and then they are substituted into eq. (4). Consequently the mechanical behavior of SMA can be estimated easily by series combined model of R-phase and austenite phase.

Accordingly mechanical properties of austenite phase element were estimated using stress-strain curve of the specimen with $\varepsilon_{\max }=0 \%$, which is considered to be a single austenite phase over the whole specimen. On the other hand, mechanical properties of R-phase element were estimated using stress-strain curve of the specimen with $\varepsilon_{\max }=10 \%$, which is considered to be a single R-phase over the whole specimen according to the discussion in the previous section. Estimated various mechanical parameters for two phases are shown in Table 2.

Next, the volume fraction of each phase with any pre-strain $\varepsilon_{\max }$ is calculated. Pre-strain corresponding to R-phase's emergence in the specimen after $400^{\circ} \mathrm{C}$ heat-treatment is defined as $\varepsilon_{\mathrm{st}}$, and pre-strain corresponding to R-phase's propagation finish over the whole specimen is defined as $\varepsilon_{\text {end }}$. On the premise that volume fraction of each phase changes

Table 2 Parameter values for the approximation.

\begin{tabular}{cccc}
\hline Phase & Parameters & Value & Unit \\
\hline & $E$ & 19.0 & $\mathrm{GPa}$ \\
Austenite & $\sigma_{\max }$ & 685 & $\mathrm{MPa}$ \\
$\left(\varepsilon_{\max }=0 \%\right)$ & $\sigma_{y}$ & 310 & $\mathrm{MPa}$ \\
& $\varepsilon_{\mathrm{el}}$ & 0.0163 & - \\
$\varepsilon_{\text {ip }}$ & 0.175 & - \\
\hline Phase & $n$ & 3 & - \\
\hline & Parameters & Value & Unit \\
\hline Rhombohedral & $E$ & 9.6 & $\mathrm{GPa}$ \\
$\left(\varepsilon_{\max }=10 \%\right)$ & $\sigma_{\max }$ & 1000 & $\mathrm{MPa}$ \\
& $\sigma_{y}$ & 215 & $\mathrm{MPa}$ \\
& $\varepsilon_{\mathrm{el}}$ & 0.0224 & - \\
& $\varepsilon_{\text {ip }}$ & 0.132 & - \\
\hline & $n$ & 3 & -
\end{tabular}


Table 3 Relation between $\varepsilon_{\max }$ and $\mathrm{R}$ phase, Austenite phase rate.

\begin{tabular}{lcc}
\hline$\varepsilon_{\max } /-$ & $p(\mathrm{R}$ phase rate $)$ & $1-p$ (Austenite phase rate) \\
\hline 0.015 & 0.00 & 1.00 \\
0.05 & 0.41 & 0.59 \\
0.08 & 0.77 & 0.23 \\
0.10 & 1.00 & 0.00 \\
0.12 & 1.00 & 0.00 \\
\hline
\end{tabular}

proportionally against pre-strain in the range between $\varepsilon_{\mathrm{st}}$ and $\varepsilon_{\text {end }}$, the volume fraction of R-phase, $p$ for pre-strain $\varepsilon_{\max }$ is expressed as eq. (9).

$$
p=\frac{1}{\varepsilon_{\mathrm{end}}-\varepsilon_{\mathrm{st}}} \varepsilon_{\mathrm{max}}-\frac{\varepsilon_{\mathrm{st}}}{\varepsilon_{\mathrm{end}}-\varepsilon_{\mathrm{st}}} .
$$

Pre-strain corresponding to R-phase's emergency in the material was set to $1.5 \%$, that is, the strain for starting twin deformation. Although stress-induced martensite transformation finishes at $12 \%$ strain, pre-strain corresponding to finish of R-phase's propagation over the whole specimen was set to $10 \%$. This is decided from facts that R-phase appeared even in noSIM area of $\varepsilon_{\max }=10 \%$ specimen and that both mechanical behaviors in $\varepsilon_{\max }=10 \%$ specimen and $\varepsilon_{\max }=$ $12 \%$ specimen are almost the same. Volume fractions of both phases for several representative pre-strains are shown in Table 3.

Using parameter values in Table 2 and volume fractions in Table 3, mechanical behaviors simulated in the case of $\varepsilon_{\max }=5 \%$ and $\varepsilon_{\max }=8 \%$ are shown in Figs. 15 and 16, respectively. From these figures, it is recognized that the simulated curve agrees well with experimental data except below $200 \mathrm{MPa}$ stress.

Therefore we can summarize from the simulation results as follows.

(1) It is correct that pre-strain corresponding to R-phase's propagation finish over the whole specimen (volume fraction of R-phase is $100 \%$ ) is around $\varepsilon_{\max }=10 \%$.

(2) In the case with pre-strain before the stress-induced martensite transformation propagates whole specimen, mechanical behavior of the whole specimen can be estimated using series combined model of R-phase and austenite phase.

In addition to that, the most significant point is that mechanical properties of both R-phase and austenite phase for simulation are independent of pre-strain's value. Up to $12 \%$ pre-strain, at which stress-induced martensite transformation propagates over the whole specimen, the constant mechanical properties of both phases can be used for simulation regardless of the position in the specimen and of pre-strain's value.

3.4.2 Mechanical properties in the case of $\varepsilon_{\max }>12 \%$

Here the mechanical properties of the specimens above $12 \%$ pre-strain are discussed. First various parameters in eqs. (5) and (6) were defined based on the experimental data of stress-strain curves shown in Fig. 12. The trends of various parameters are shown in Figs. 17-19 as a function of pre-strain.

Figure 17 shows relationship between Young's modulus E and pre-strain $\varepsilon_{\max }$, and Fig. 18 shows plots of yield stress $\sigma_{y}$

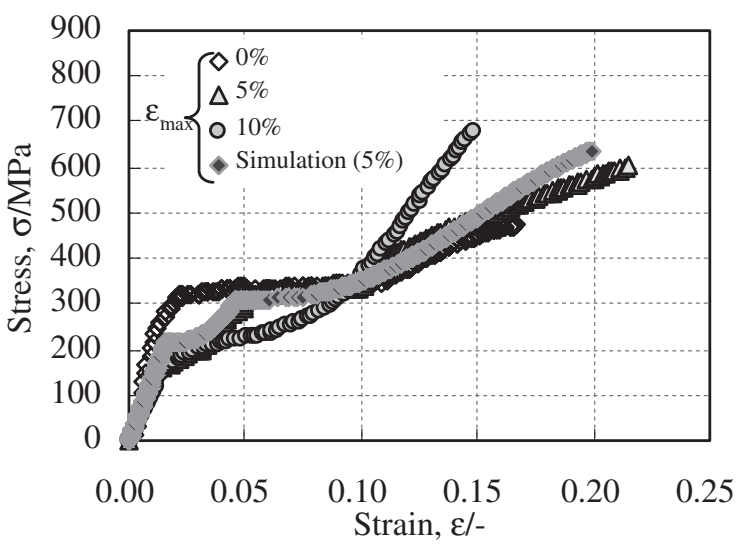

Fig. 15 Stress-strain behavior of $\varepsilon_{\max }=5 \%$ in comparison with the simulation.

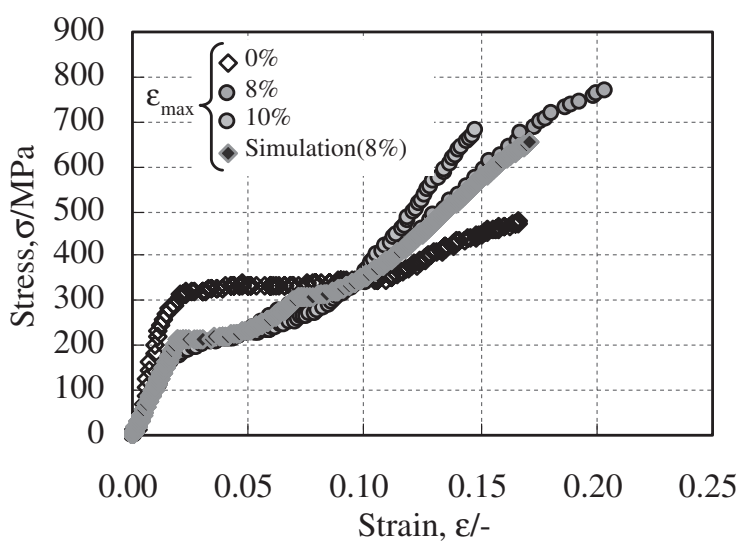

Fig. 16 Stress-strain behavior of $\varepsilon_{\max }=8 \%$ in comparison with the simulation.

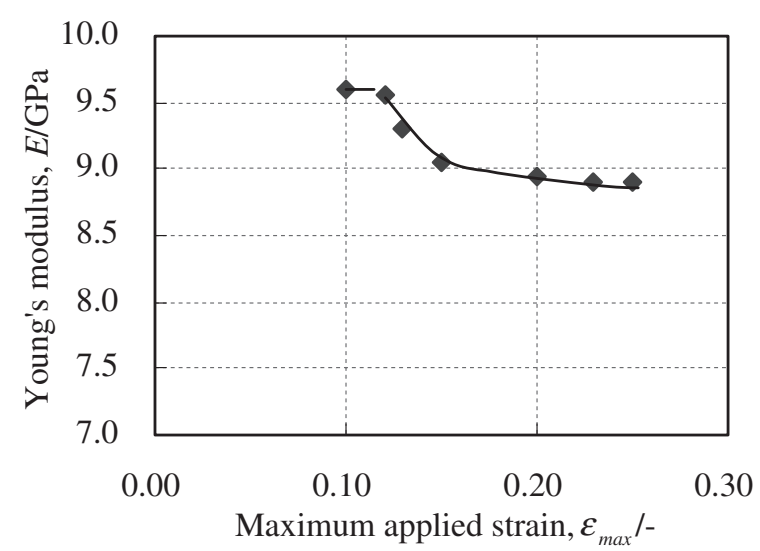

Fig. 17 Relationship between maximum applied strain $\varepsilon_{\max }$ and young's modulus $E$.

against pre-strain $\varepsilon_{\max }$. Additionally Fig. 19 shows plots of strain corresponding to the inflection point of a stress-strain curve against pre-strain $\varepsilon_{\max }$. Parameter $n$ is set to 3 for all pre-strain.

From these figures, it is found that Young's modulus decreases with increasing pre-strain as well as yield stress. The yield stress corresponds to a stress at which twin reorientation starts. As the crystal orientation in the specimen 


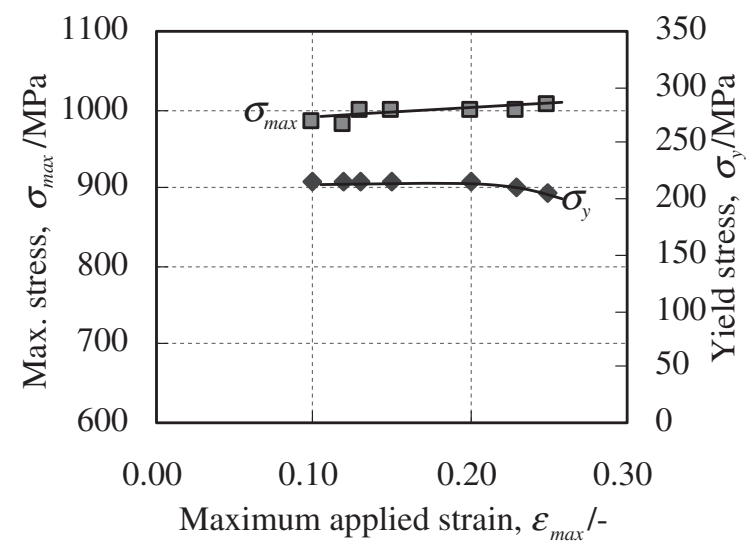

Fig. 18 Relationship among maximum applied strain $\varepsilon_{\max }$, maximum stress $\sigma_{\max }$, and yield stress $\sigma_{y}$.

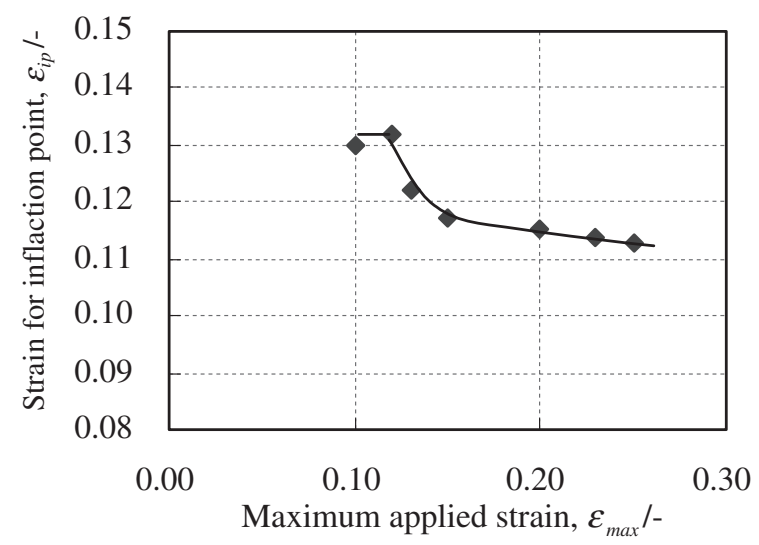

Fig. 19 Relationship between maximum applied strain $\varepsilon_{\max }$ and strain for the inflection point $\varepsilon_{\text {ip }}$.

begins to arrange in a constant direction with increasing prestrain, the deformation resistance is considered to get lower. This is the reason why the Young's modulus and yield stress decrease with increasing pre-strain. On the other hand, although the maximum stress $\sigma_{\max }$ slightly increases with increasing pre-strain, the strain $\varepsilon_{\text {ip }}$ concerning inflection point of the curve decreases with increasing pre-strain. The latter fact means that a stress-strain curve in the second deformation stage corresponding to eq. (6) shift to the left on the stress-strain diagram. These results show that the deformation progresses in lower stress as pre-strain gets larger. On the other hand, as pre-strain gets larger, the strain for finishing twin reorientation becomes gradually smaller and the deformation resistance above the strain gets higher.

Relationship between stress and strain can be expressed as a function of some parameters because the parameters are expressible as a function of pre-strain as shown in Figs. 1719. Thus, mechanical behavior of the specimen with prescribed pre-strain can be estimated by eqs. (10) and (11).

$$
\begin{gathered}
\sigma=\hat{E} \varepsilon \\
\sigma=\left(\hat{\sigma}_{\max }-\hat{\sigma}_{y}\right)\left[1-\exp \left\{-\hat{\varepsilon}_{\mathrm{el}}\right)\right. \\
\left.\left.\left(\frac{\varepsilon-\hat{\varepsilon}_{\mathrm{el}}}{\hat{\varepsilon}_{\mathrm{ip}}}\right)^{n}\right\}\right]+\hat{\sigma}_{y} \\
\left(\varepsilon \geq \hat{\varepsilon}_{\mathrm{el}}\right)
\end{gathered}
$$

$n$ is 3 for all pre-strain, and parameters with hat symbol, “^” indicate they are function of pre-strain $\varepsilon_{\max }$. These expressions are in the range of $0.12 \leq \varepsilon_{\max }<0.25$.

\section{Conclusions}

Thin tubes of shape memory alloy with the composition of Ti-51 at $\% \mathrm{Ni}$ subjected to $20 \%$ cold working were used for this study. Test specimens were made of rectangular thin plates cut out from the tubes after annealing at $600^{\circ} \mathrm{C}$ for $1 \mathrm{~h}$.

The influences of pre-strain and subsequent heat-treatment on mechanical properties were investigated for specimens with applied pre-strain and for specimens with added heattreatment at $400^{\circ} \mathrm{C}$ for $1 \mathrm{~h}$. The results can be summarized as follows.

(1) With respect to the specimens after annealed at $600^{\circ} \mathrm{C}$ for $1 \mathrm{~h}$ in the atmosphere, it is possible to identify whether stress-induced martensite transformation and subsequent twin deformation occur or not by observing oxidized coating peeled off from the substrate during tensile tests.

(2) The difference between martensite start temperature $\left(M_{\mathrm{s}}\right)$ and martensite finish temperature $\left(M_{\mathrm{f}}\right)$ of the area, which has experienced stress-induced martensitic transformation (SIM) and subsequent twin deformation, is bigger than that of the area which has not experienced them (noSIM) yet. This fact is due to the internal stress fields generated among the crystals in the twin deformation process.

(3) As for the area that has experienced stress-induced martensite transformation and subsequent twin deformation, R-phase emerges surely in the microstructural phase after annealed at $400^{\circ} \mathrm{C}$ for $1 \mathrm{~h}$. Concerning the area that has not experienced stress-induced martensite transformation, R-phase can appear in small area near the borderline between SIM area and noSIM area.

(4) Relation between stress and strain of the specimens after annealed at $400^{\circ} \mathrm{C}$ for $1 \mathrm{~h}$ was discussed in two cases according to pre-strain before the heat-treatment. Until reaching the $12 \%$ pre-strain, at which stress-induced martensite transformation finishes to propagate over the whole specimen, the relation between stress and strain can follow well the series combined model of R-phase and austenite phase. On the other hand, above $12 \%$ pre-strain, the relation can be expressed by the exponential function as a parameter of pre-strain.

\section{REFERENCES}

1) K. Tanaka, H. Tobushi and S. Miyazaki: Mechanical properties of Shape Memory Alloys, (Yokendo Ltd., 1993) pp. 7-61.

2) J. I. Kim, Y. Liu and S. Miyazaki: Acta Mater. 52 (2004) 487-499.

3) Y. Liu, H. Yang and A. Voigt: Mater. Sci. Eng. A 360 (2003) 350-355.

4) P. Filip and K. Mazanec: Scr. Mater. 45 (2001) 701-707.

5) H. Semba, N. Okabe, T. Yamaji, K. Okita and K. Yamauchi: Mater. Sci. Forum 475-479 (2005) 2055-2058.

6) H. Semba, N. Okabe, T. Yamaji, K. Okita and K. Yamauchi: Mater. Sci. Forum 475-479 (2005) 2059-2062.

7) M. Dolce and D. Cardone: Int. J. Mechanic. Sci. 43 (2001) 2631-2656.

8) M. Dolce and D. Cardone: Int. J. Mechanic. Sci. 43 (2001) 2657-2677.

9) N. Okabe and M. Hosogi: Science of machine, (Yokendo Ltd.) 54 (2002) 941-946. 